\title{
ESTIMATION OF DOUBLE REDUCTION AND GENETIC PARAMETERS IN AUTOTETRAPLOIDS BASED ON 4X-2X AND 4X-4X MATINGS
}

\author{
G. C. C. TAI \\ Research Station, Agriculture Canada, Fredericton, N.B., and Department of Genetics, \\ University of Birmingham, Birmingham, U.K.
}

Received 1.vii.82

\section{SUMMARY}

A mating scheme is proposed for estimating double reduction coefficient and genetic parameters in an additive-dominance model for autotetraploids. The scheme uses three parents in two $4 \mathrm{X}-2 \mathrm{X}$ and four $4 \mathrm{X}-4 \mathrm{X}$ matings. The parents are a homozygous tetraploid $(A A A A)$, a diploid hybrid $(A a)$ and its tetraploid $(A A a a)$. The usefulness of the scheme is compared with the one proposed by Tai (1982).

\section{INTRODUCTION}

A METHOD was proposed by Tai (1982) for estimating double reduction and genetic parameters in an additive-dominance model for autotetraploids. It used a diploid hybrid $A a$ and its tetraploid in a series of seven crosses. The diploid hybrid produces $2 n$ unreduced gametes by either first or second division restitution during meiosis. There are three types of crosses involved in the mating design, i.e., diploid $\times$ diploid $(2 X-2 X)$, diploid $\times$ tetraploid $(2 X-4 X)$ and tetraploid $\times$ tetraploid $(4 X-4 X)$. The use of $2 X-2 X$ cross in the mating system requires that the gametic output in $2 n$ pollen is the same as that in $2 n$ eggs. Experimental work in potato (Mendiburu and Peloquin 1971) indicates that there is no difficulty in obtaining $4 X$ progenies from $2 X-2 X$ matings. Most cytogenetic and genetic studies on $2 n$ gametes (e.g., Bingham, 1968; Mok and Peloquin, 1975a,b; Ramanna, 1979) tend to concentrate on $2 n$ pollen and its usefulness in plant breeding. No evidence is available to indicate whether the mechanisms of $2 n$ pollen and egg production are identical in a diploid individual. Bias would be introduced into Tai's estimates of various parameters if the mechanism of $2 n$ gamete production is somewhat different between male and female gametophytes.

The purpose of the present paper is to propose an alternative mating scheme for estimating the double reduction coefficient and genetic parameters of autotetraploids. It uses a homozygous tetraploid, a diploid hybrid and its tetraploid in a mating scheme involving six crosses. Only two types of crosses, i.e., $4 X-2 X$ and $4 X-4 X$, are used in the system. The diploid parent can be solely used as male or female parent in the crosses. The problem of having identical gametic output in $2 n$ pollen and egg is thus avoided.

\section{MAting SChEME}

The mating scheme involves three parents: a diploid hybrid $(A a)$, its tetraploid ( $A A a a$ or $A_{2} a_{2}$ ) and a homozygous tetraploid $\left(A A A A\right.$ or $\left.A_{4}\right)$. 
The diploid hybrid is obtained by crossing the haploid $(\boldsymbol{A} A)$ extracted from $A_{4}$ with a haploid derived from another tetraploid or diploid genotype from another species. The three parents are referred to as $P_{1}, P_{2}$ and $P_{3}$. Six crosses are made between them:

$\begin{array}{ccc}\text { Type of crosses } & \text { Mating scheme } & \text { Symbol } \\ 4 X \times 2 X & A_{2} a_{2} \times A a & \mathrm{~F}_{12} \\ 4 X \times 4 X & A_{2} a_{2} \times A_{2} a_{2} & \mathrm{~F}_{22} \\ 4 X \times 2 X & A_{4} \times A a & \mathrm{~F}_{13} \\ 4 X \times 4 X & A_{4} \times A_{2} a_{2} & F_{23} \\ 4 X \times 4 X & A_{4} \times\left(A_{4} \times A a\right) & \mathrm{B}_{3(13)} \\ 4 X \times 4 X & A_{4} \times\left(A_{4} \times A_{2} a_{2}\right) & \mathrm{B}_{3(23)}\end{array}$

It is noted that the diploid parent $(A a)$ can be restricted as male( or female) parent in the two $4 X-2 X$ crosses if required. It produces $2 n$ pollen by first (FDR) and/or second (SDR) division restitution during meiosis. The method of estimation using the FDR diploid parent will be described in the following section.

\section{Method of estimation}

The additive-dominance model (Mather and Jinks, 1971) defines the five possible tetraploid genotypes as having different mean phenotypic deviations from $m$, the mid-homozygote value, as follows: $A_{4}, d ; A_{3} a, h_{3}$; $A_{2} a_{2}, h_{2} ; A a_{3}, h_{1} ;$ and $a_{4},-d$. The expected mean of a cross can then be expressed as

$$
\text { Mean }=m+c_{1} d+c_{2} h_{3}+c_{3} h_{2}+c_{4} h_{1}
$$

where the $c$ 's are coefficients associated with the genetic parameters $d, h_{3}$, $h_{2}$ and $h_{1}$ in a cross. To determine the sizes of the $c$ 's we need to know the gametic output of the five tetraploid and FDR diploid $(A a)$ genotypes. Let $\alpha$ be the frequency of double reduction in the tetraploid (Fisher and Mather, 1943) and $\beta$ that of single exchange tetrads in the diploid (Mendiburu and Peloquin, 1979; Tai, 1982). Also denote $u_{1}=(2+\alpha) / 4$, $u_{2}=(1-\alpha) / 2, u_{3}=\alpha / 4, u_{4}=(1+2 \alpha) / 6, u_{5}=(2-2 \alpha) / 3, v_{1}=\beta / 4$ and $v_{2}=$ $(2-\beta) / 2$. The gametic frequencies of five tetraploids and the FDR diploid can be expressed in terms of the $u$ 's and $v$ 's as shown in table 1 . Using the information in table 1 , the $c$ coefficients in the expected means of $P_{2}, P_{3}$ and six crosses in the mating scheme are given in table 2.

\section{TABLE 1}

Gametic output of five autotetraploids and a FDR diploid

$\begin{array}{cccc}\text { Parental } \\ \text { genotype } & \overbrace{\boldsymbol{A A}} & \boldsymbol{A a} & a a \\ \boldsymbol{A}_{4} & 1 & - & - \\ \boldsymbol{A}_{3} a & u_{1} & u_{2} & u_{3} \\ \boldsymbol{A}_{2} a_{2} & u_{4} & u_{5} & u_{4} \\ \boldsymbol{A} a_{3} & u_{3} & u_{2} & u_{1} \\ a_{4} & - & - & 1 \\ A a \text { (FDR) } & v_{1} & v_{2} & v_{1}\end{array}$


TABLE 2

Coefficients associated with genetic parameters in the expressions of expected means of $P_{2}$ and $P_{3}$ and six crosses in the mating scheme

\begin{tabular}{lccccc}
\multicolumn{5}{c}{ Genetic parameters } \\
\cline { 2 - 6 } Means & $m$ & $d$ & $h_{3}$ & $h_{2}$ & $h_{1}$ \\
$\bar{P}_{2}$ & 1 & 0 & 0 & 1 & 0 \\
$\bar{P}_{33}$ & 1 & 1 & 0 & 0 & 0 \\
$\bar{F}_{12}$ & 1 & 0 & $u_{4} v_{2}+u_{5} v_{1}$ & $2 u_{4} v_{1}+u_{5} v_{2}$ & $u_{5} v_{1}+u_{4} v_{2}$ \\
$\bar{F}_{22}$ & 1 & 0 & $2 u_{4} u_{5}$ & $2 u_{4}^{2}+u_{5}^{2}$ & $2 u_{4} u_{5}$ \\
$\bar{F}_{13}$ & 1 & $v_{1}$ & $v_{2}$ & $v_{1}$ & 0 \\
$\bar{F}_{23}$ & 1 & $u_{4}$ & $u_{5}$ & $u_{4}$ & 0 \\
$\bar{B}_{3(13)}$ & 1 & $v_{1}+u_{1} v_{2}+u_{4} v_{1}$ & $u_{2} v_{2}+u_{5} v_{1}$ & $u_{3} v_{2}+u_{4} v_{1}$ & 0 \\
$\bar{B}_{3(23)}$ & 1 & $u_{4}+u_{1} u_{5}+u_{4}^{2}$ & $u_{2} u_{5}+u_{4} u_{5}$ & $u_{3} u_{5}+u_{4}^{2}$ & 0
\end{tabular}

The expected means of $P_{2}, P_{3}, F_{12}, F_{13}, F_{22}$ and $F_{23}$ can be easily obtained using table 1 . Those of $\mathrm{B}_{3(13)}$ and $\mathrm{B}_{3(23)}$ cannot be readily derived. The detailed derivation of $\overline{\mathrm{B}}_{3(13)}$ is given as follows:

(a) The cross $A_{4} \times A a$ produces the progenies $A_{4}, A_{3} a, A_{2} a_{2}, A a_{3}$ and $a_{4}$ with frequencies $v_{1}, v_{2}, v_{1}, 0$ and 0 , respectively.

(b) The relative frequencies of $A A, A a$ and $a a$ gametes produced by genotypes $A_{4}, A_{3} a$ and $A_{2} a_{2}$ are, respectively, $1: 0: 0 ; u_{1}: u_{2}: u_{3}$; and $u_{4}: u_{5}: u_{4}$ (table 1$)$.

(c) Backcrossing the progenies $A_{4}, A_{3} a$ and $A_{2} a_{2}$ to $P_{3}\left(A_{4}\right)$ produces the progenies $A_{4}, A_{3} a$ and $A_{2} a_{2}$ with relative frequencies: $v_{1}: 0: 0$; $u_{1} v_{2}: u_{2} v_{2}: u_{3} v_{2}$; and $u_{4} v_{1}: u_{5} v_{1}: u_{4} v_{1}$, respectively.

(d) The frequencies of $A_{4}, A_{3} a$ and $A_{2} a_{2}$ progenies in the cross $A_{4} \times$ $\left(A_{4} \times A a\right)$, i.e., $B_{3(13)}$, are consequently $v_{1}+u_{1} v_{2}+u_{4} v_{1}, u_{2} v_{2}+u_{5} v_{1}$ and $u_{3} v_{2}+u_{4} v_{1}$, respectively.

(e) The expected mean of $B_{3(13)}$ is then

$$
\bar{B}_{3(13)}=m+\left(v_{1}+u_{1} v_{2}+u_{4} v_{1}\right) d+\left(u_{2} v_{2}+u_{5} v_{1}\right) h_{3}+\left(u_{3} v_{2}+u_{4} v_{1}\right) h_{2} .
$$

The following results can be obtained from table 2 :

$$
\begin{aligned}
& y_{1}=\bar{F}_{13}-\bar{F}_{23}=\gamma\left(-d+2 h_{3}-h_{2}\right) \\
& y_{2}=6\left(\bar{B}_{3(13)}-\bar{B}_{3(23)}\right)=\gamma(1-\alpha)\left(-d+2 h_{3}-h_{2}\right) \\
& y_{3}=3\left(\bar{F}_{23}-\left(\bar{P}_{2}+\bar{P}_{3}\right) / 2\right)=(1-\alpha)\left(-d+2 h_{3}-h_{2}\right)
\end{aligned}
$$

where $\gamma=(2+4 \alpha-3 \beta) / 12=u_{4}-v_{1}=\left(v_{2}-u_{5}\right) / 2$. Thus,

$$
\hat{\alpha}=1-\left(y_{2} / y_{1}\right)
$$

and $\hat{\gamma}=y_{2} / y_{3}$.

Also, $\hat{\beta}=(2+4 \hat{\alpha}-12 \hat{\gamma}) / 3$. The $\hat{\alpha}$ and $\hat{\beta}$ values are then used to estimate the $u$ 's and $v$ 's in table 1 and the coefficients involving the $u$ 's and $v$ 's in table 2.

The genetic parameters $m, d, h_{3}, h_{2}$ and $h_{1}$ are estimated by a weighted least squares procedure (Mather and Jinks, 1971). The weights are the reciprocal of the squared standard errors for $\overline{\mathrm{P}}_{2}, \overline{\mathrm{P}}_{3}$ and six cross means. Let $V$ be the diagonal matrix of squared errors for the means, $O$ the vector 
of observed means, and $\hat{M}$ the vector of estimates of genetic parameters,

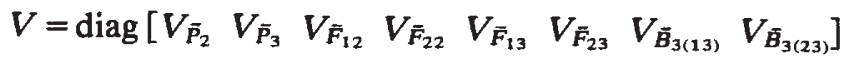

$$
\begin{aligned}
& O=\left[\begin{array}{l}
\bar{P}_{2} \\
\bar{P}_{3} \\
\bar{F}_{12} \\
\bar{F}_{22} \\
\bar{F}_{13} \\
\bar{F}_{23} \\
\bar{B}_{3(13)} \\
\bar{B}_{3(23)}
\end{array}\right] \quad \hat{M}=\left[\begin{array}{l}
\hat{m} \\
\hat{d} \\
\hat{h}_{3} \\
\hat{h}_{2} \\
\hat{h}_{1}
\end{array}\right]
\end{aligned}
$$

Also let $C$ be the matrix of $c$ coefficients as given in table 2. We have

$$
M=\left(C^{\prime} V^{-1} C\right)^{-1} C^{\prime} V^{-1} O
$$

where $C^{\prime}$ is the transpose of $C$.

The estimates of genetic parameters can then be used in a joint scaling test for goodness of fit of the additive-dominance model (Mather and Jinks, 1971). The $\chi^{2}$ statistic has one degree of freedom since seven parameters (i.e., $m, \alpha, \beta, d, h_{3}, h_{2}$ and $h_{1}$ ) are fitted out of eight observed means.

\section{Discussion}

The mating scheme of Tai (1982) uses a diploid hybrid $(A a)$ and its tetraploid $\left(A_{2} a_{2}\right)$ as parents. It does not need a homozygous tetraploid parent as required in the present system. It thus has advantages for investigating an asexually propagated species such as the potato since the available tetraploid clones are usually heterozygous. The present system, while requiring additional time and effort to self heterozygous tetraploids for parental material, is more reliable for use when no assurance can be given that the mechanisms of $2 n$ sporogenesis in male and female gametophytes are the same in a diploid parent.

A faster way to obtain homozygous materials would be at the diploid rather than at the tetraploid level. Thus, an haploid population can be created at first from heterozygous tetraploid clones. Continuous selfing followed by chromosome doubling would give materials to start a mating scheme.

The mating scheme proposed here uses the minimum number of crosses required for estimating the various parameters. Precision of estimation can be increased when more generations are involved in the mating design. For example, the backcross generations $B_{1(12)}, B_{2(12)}, B_{1(22)}$ and $B_{2(22)}$ which are used in the mating scheme of Tai (1982) can be added to the present system.

The method of estimation is illustrated by using a FDR diploid parent. As indicated by Tai (1982), the same principle applies when a diploid parent produces $2 n$ gametes by SDR or a mixture of FDR and SDR. 
Acknowledgements. - The work was conducted while I was on educational leave supported by Agriculture Canada. I am grateful to Professor J. L. Jinks for his encouragement and critical reading of the paper.

\section{REFERENCES}

BINGHAM, E. T. 1968. Transfer of diploid medicago spp. germplasm to tetraploid $M$. sativa L. in $4 \mathrm{X}-2 \mathrm{X}$ crosses. Crop. Sci., 8, 760-762.

FISHER, R. A., AND MATHER, K. 1943. The inheritance of style length in Lythrum salicaria. Ann. Eugenics, 12, 1-23.

MATHER, K., AND JINKS, J. L. 1971. Biometrical Genetics. Second edition. Chapman Halt, London.

MENDIBURU, A. O., AND PELOnJIN, S. J. 1971. High yielding tetraploids from 4X-2X and 2X-2X matings. Am. Potato J., 48, 300-301.

MENDIBURU, A. O., AND PELOQUIN, S. J. 1979. Gene-centrometre mapping by $4 \mathrm{X}-2 \mathrm{X}$ matings in potatoes. Theor. Appl. Genet., 54, 177-180.

MOK, D. W. S., AND PELOQUIN, S. J. $1975 a$. The mechanisms of $2 n$ pollen formation in diploid potatoes. Can. J. Genet. Cytol., 17, 217-212.

MOK, D. W. S., AND PELOQUIN, S. J. 1975b. The inheritance of three mechanisms of diplandroids $(2 n$ pollen) formation in diploid potatoes. Heredity, 35, 295-302.

RAMANNA, M. S. 1979. A re-examination of the mechanisms of $2 n$ gamete formation in potato and its implications for breeding. Euphytica, 28, 537-561.

TAI, G. C. C. 1982. Estimation of double reduction and genetic parameters in autotetraploids. Heredity, 49, 63-70. 